\section{PERFORATION OF THE STOMACH.}

\author{
To the Editor of THE LANCET.
}

SIR,-I am induced to forward the enclosed most interesting case, from the importance of the subject rendering the recording of simple facts more valuable than the most elaborate description of disease. Oculis subjectafidelis. I am, Sir, your obedient servant,

\section{Ramsgate, July 23, 1842.}

G. Friend Whiteley.

At eleven o'clock, p.m., July 14th, M. B., a lady's-maid, aged 26, of interesting and prepossessing appearance, with pale complexion, was seized whilst ascending the stairs with what she described as a spasm, referrible to the stomach. She had been subject to occasional pain in that viscus, as also in both hypochondria, but was always soon relieved by medical treatment. I saw her immediately after the attack, and found her on the floor suffering agonising pains in the whole abdomen. They seemed to originate from the scrobiculis cordis, and extended to the hypochondrium and back. She had taken tea at five o'clock, apparently in good spirits, and without any disposition to vomit or feeling of uneasiness. Her countenance now was exceedingly anxious; pulse not much hurried; great dread of taking a deep inspiration; tongue clean; skin natural; the bowels had been inactive for two days. Urdered-

\section{Castor-oil, $\overline{3}$ ss ;}

Tincture of opium, m. $\mathrm{xxx}$.

To be taken immediately. A fomentation of poppies to be applied to the seat of pain.

Twelve o'clock. The pain more urgent; skin hot; pulse increased; no action of the bowels. Warm water enema.

Re Calomel, gr. v; Opium, gr. i.

To be taken every two hours.

Two o'clock. The pain still very severe; unable to bear the least pressure of the hand; the sitting posture seemed the easiest. The medicines had been vomited. Ordered to be bled to eighteen ounces in full stream. Repeat the fomentations.

Three o'clock. Pain continues excessive; pulse 120, small and rumning; a quantity of dark grumous fluid was ejected from the stomach; the countenance slightly changed; skin bedewed with moisture; bowels motionless. Eighteen leeches to the affected region.

\section{R Croton-oil, gtts. iss;} Tincture of opium, $3 \mathrm{i}$.

Continue the fomentation.

Five o'clock. No abatement of the pain. I now called in my friend Dr. Canham, who ordered an enema, consisting of two pounds of gruel and an ounce of castor-oil, to be given immediately.

No. 988 .
R Calomel, gr. viij ;

Opium, gr. iv;

Compound extract of colocynth, $\mathrm{gr} . \mathbf{x v}$. Make into eight pills, of which let two be taken every other night.

The injection caused no pain.

Eight o'clock. The patient is reported to have slept half an hour; about six ounces of the injection have been evacuated, unaccompanied with the least freal odour; pain still the same; pulse weaker. Colocynth enema ; omit the pills; effervescing draught.

Ten o'clock. The patient seemed revived, and drank fieely of the effervescing draught, but said she was enduring much pain. We now left her, as she seemed inclined to doze, and intended to have called again in two hours. At eleven o'clock $I$ was sent for, and on entering the apartment perceived her dissolution evidently approaching; her respiration was short and difficult; extremities cold ; face livid; pulse hardly perceptible; there was apparently no intermission of the pain, and having had an involuntary discharge of fæces, she expired at half-past eleven o'clock.

\section{Autopsy Tuenty-six Hours after Death.}

The muscular system was firm and healthy, with a due proportion of adipose sub. stance ; intestines distended with flatus, and the injections, and having a slight blush of inflammation. On exposing the stomach a perforation, the effect of ulceration, was discovered (situated on the anterior surface at the union of the pyloric with the cardiac portions). The ulcer, about the size of a fourpenny-piece, hollow, rather oval in shape, thin and shining, with callous edges. The stomach contained merely a dark-biown fluid, but was highly vascular, especially in the neighbourhood of the ulcer. The other organs of the body healthy.

The points which command attention in this instructive case are-

1. The suddenness of the attack.

2. The pain characterised by its severity and continuance without any alleviation.

3. The natural state of the pulse for some time after the attack.

4. The rapid sinking of the powers of life.

Accidents in Mrnes.-Dr. Barham had had returns from the majority of all the mines in Devon and Cornwall, of accidents in them during the last two years. From these returus, he found that the whole number of underground miners amounted to 12,675. The number of accidents within the last two years had been 75 ; or a proportion of one in 338. The per-centage of deaths from mine accidents, stated last year to have occurred in the district comprising Gwennap, Illogan, and Clamborne, was 17 3-10ths per cent. on the whole number of deaths in the district.-Annual Report of the Cornwall Polytechnic Society. 